\title{
Underground Nuclear Astrophysics: Present and future of the LUNA experiment
}

\author{
Carlo Gustavino ${ }^{a}$ \\ ${ }^{1}$ INFN Sezione di Roma, I-00185 Roma, Italy
}

\begin{abstract}
The evolution of celestial bodies is regulated by gravitation and thermonuclear reaction rates, while the Big Bang nucleosynthesis is the result of nuclear processes in a rapidly expanding Universe. The LUNA Collaboration has shown that, by exploiting the ultra low background achievable deep underground, it is possible to study the relevant nuclear processes down to the nucleosynthesis energy inside stars and during the first minutes of Universe. In this paper the main results of LUNA are overviewed, as well as the scientific program the forthcoming 3.5 MV underground accelerator.
\end{abstract}

\section{Introduction}

The synthesis of elements in stars are due to thermonuclear fusion reactions, which start from the most abundant and lightest element, the hydrogen, towards to synthesis of the heaviest elements. Therefore nuclear fusion reactions determine the energy production, the neutrino luminosity and the evolution of stars. In the stellar environment the thermal energy of nuclei is much lower than the Coulomb barrier. As an example, the core temperature of the Sun is about $15 \cdot 10^{6} \mathrm{~K}$, corresponding to a Maxwell-Boltzmann energy distribution peaked to about $1 \mathrm{keV}$, much lower than the $550 \mathrm{keV}$ effective Coulomb barrier for the $p+p$ reaction. Indeed, the nuclear reactions take place via quantum tunneling effect and the cross section of charged particle reactions can be expressed as follows:

$$
\sigma(E)=\frac{S(E) e^{-2 \pi \eta}}{E}
$$

In this formula, the exponential term takes into account the Coulomb barrier, while the astrophysical factor $S(E)$ contains all the nuclear effects. For non-resonant reactions, it is a smoothly varying function of energy. The Sommerfeld parameter $\eta$ is given by $2 \pi \eta=31.29 Z_{1} Z_{2}(\mu / E)^{1 / 2} \cdot Z_{1}$ and $Z_{2}$ are the nuclear charges of the interacting nuclei. $\mu$ is their reduced mass (in units of a.m.u.), and $E$ is the center of mass energy (in units of $\mathrm{keV}$ ) [1,2].

Fusion reactions in stars occur in a well defined energy range, the so called Gamow peak, which arises from the convolution of the thermal energy distribution of nuclei and the tunneling probability through the Coulomb barrier between the interacting nuclei. For a given stellar temperature $\mathrm{T}$ the energy corresponding to the Gamow peak is larger than KT, typically in the order of tens of keV. Most of the reactions between light nuclei at such energies have cross sections of the order of $10^{-9} \div 10^{-12}$ barn.

ae-mail: carlo.gustavino@roma1.infn.it 
Table 1. Selection of nuclear reaction already studied by LUNA (a), in progress (b) and in program (c).

\begin{tabular}{|c|c|c|c|}
\hline Reaction & main motivation & accelerator & reference \\
\hline${ }^{3} \mathrm{He}\left({ }^{3} \mathrm{He}, 2 \mathrm{p}\right){ }^{4} \mathrm{He}^{a}$ & p-p chain & "LUNA-50 & [4] \\
\hline$d\left({ }^{3} H e, p\right)^{4} H e^{a}$ & electron screening & LUNA-50 & {$[8--10]$} \\
\hline $\mathrm{d}(\mathrm{p}, \gamma)^{3} \mathrm{He}^{a, b}$ & $\mathrm{BBN}$ & LUNA-50, LUNA-400 & {$[11--13]$} \\
\hline $3 \mathrm{He}(\alpha, \gamma)^{7} \mathrm{Be}^{a}$ & p-p chain, BBN & LUNA-400 & {$[14,15]$} \\
\hline${ }^{14} \mathrm{~N}(\mathrm{p}, \gamma){ }^{15} \mathrm{O}^{a}$ & CNO cycle I & LUNA-400 & {$[16--20]$} \\
\hline${ }^{25} \mathrm{Mg}(\mathrm{p}, \gamma)^{26} \mathrm{Al}{ }^{a}$ & Mg-Al cycle & LUNA-400 & {$[21--23]$} \\
\hline${ }^{15} \mathrm{~N}(\mathrm{p}, \gamma){ }^{16} \mathrm{O}^{a}$ & CNO cycle II & LUNA-400 & {$[24,25]$} \\
\hline${ }^{17} \mathrm{O}(\mathrm{p}, \gamma){ }^{18} \mathrm{~F}^{a}$ & CNO cycle III & LUNA-400 & {$[26,27]$} \\
\hline${ }^{22} \mathrm{Ne}(\mathrm{p}, \gamma)^{23} \mathrm{Na}^{a}$ & $\mathrm{Ne}-\mathrm{Na}$ cycle & LUNA-400 & {$[28,29]$} \\
\hline${ }^{17} \mathrm{O}(\mathrm{p}, \alpha){ }^{14} \mathrm{~N}^{a}$ & CNO cycle III & LUNA-400 & {$[30,31]$} \\
\hline $\mathrm{d}(\alpha, \gamma)^{6} \mathrm{Li}^{a}$ & $\mathrm{BBN}$ & LUNA-400 & {$[32,33]$} \\
\hline${ }^{13} \mathrm{C}(\alpha, n){ }^{16} \mathrm{O}^{b, c}$ & S-process & LUNA-400, LUNA-MV & {$[12,34]$} \\
\hline${ }^{22} \mathrm{Ne}(\alpha, n)^{25} \mathrm{Mg}^{c}$ & S-process & LUNA-MV & [34] \\
\hline${ }^{12} \mathrm{C}+{ }^{12} \mathrm{C}$ reactions ${ }^{c}$ & Carbon burning & LUNA-MV & [34] \\
\hline
\end{tabular}

These correspond, under typical laboratory conditions, to experimental counting rates ranging from a few events per day to a few events per month. This means that in realistic experimental conditions, the expected counting rate is prohibitively low with respect to the detector background induced by the cosmic rays. A drastic reduction of this background can be achieved by carrying out the measurement deep underground. The "Laboratori Nazionali del Gran Sasso" (LNGS) hosts the facility LUNA (Laboratory for Underground Nuclear Astrophysics), that is still the worldwide unique facility operating deep underground, in which cross sections at energies of astrophysical interest are measured. The average depth of $1400 \mathrm{~m}$ of rock leads to a reduction of the muon and neutron fluxes by a factor $10^{6}$ and $10^{3}$, respectively. The suppression of the cosmic ray induced background also allows an effective suppression of the $\gamma$-ray activity by a factor $10^{3} \div 10^{5}$, depending on the photon energy.

\section{The LUNA experiment}

High beam current, long-term stability, and precise beam-energy determination are the requirements for an ion accelerator conceived for direct nuclear astrophysics measurements. The first feature maximizes the reaction rate, the second provides the long time typically needed for a cross-section measurement, and the third is important because of the exponential energy dependency of the cross section (see eq. 1).

The first accelerator used at LUNA was a $50 \mathrm{kV}$ machine, consisting of a duoplasmatron ion source, an ion-beam extraction and acceleration system, and a double-focusing $90^{\circ}$ analyzing magnet. This machine was designed to deliver beams of protons, ${ }^{3} \mathrm{He}$ and ${ }^{4} \mathrm{He}$ ions of about $400 \mu \mathrm{A}$ at energies between 10 and $50 \mathrm{keV}$ with an energy spread of less than $20 \mathrm{eV}$ and a long-term stability [3]. With this accelerator have been measured, for the first time inside the solar Gamow peak, the cross section of the ${ }^{3} \mathrm{He}\left({ }^{3} \mathrm{He}, 2 \mathrm{p}\right){ }^{4} \mathrm{He}$ reaction [4]. The achievement of measuring the ${ }^{3} \mathrm{He}\left({ }^{3} \mathrm{He}, 2 \mathrm{p}\right){ }^{4} \mathrm{He}$ cross section inside the solar Gamow peak can be considered a milestone in the long way to the discovery of neutrino oscillation [5,6]. In fact the existence of the Fowler resonance to explain the "Solar neutrino problem" was definitively rejected, pushing towards a new generation of experiments addressed to measure the neutrino oscillation parameters and the solar interior (Borexino, Kamland, SNO). It is 
worth to point out that at the lowest energy of $\mathrm{E}_{c m}=16.5 \mathrm{keV}$ the measured cross section is $0.02 \pm 0.02$ $\mathrm{pb}$, which corresponds to a rate of about 1 event/month, rather low even for the "silent" experiments of underground physics [4]. Even though the LUNA-50 accelerator has produced outstanding results, it should be considered as a pilot project toward the present facility, the LUNA-400 accelerator [7]. As it is summarised in table 1, the LUNA-400 accelerator is mainly employed to study reactions of hydrogen burning in the Sun and in other celestial bodies, e.g. massive stars, AGB stars, Novae. Also important is the study of several leading processes of Big Bang Nucleosynthesis (BBN). As an example, the ongoing study of the $\mathrm{d}(\mathrm{p}, \gamma)^{3} \mathrm{He}$ reaction is particularly important to derive the cosmological baryon density $\Omega_{b}$ and to constrain the possible existence of "dark radiation" at BBN epoch, i.e. the existence of light particles such as sterile neutrinos or axions, not foreseen in the standard model.

Although the LUNA-400 accelerator is presently full operative, the future of LUNA is addressed to study the successive phases of stars, towards the synthesis of heavier and heavier elements. In this concern the LUNA-MV project has been approved and funded, and will be operative in 2019. This machine has a terminal Voltage of 3.5 MV and can deliver beams of ${ }^{1} \mathrm{H}^{+},{ }^{4} \mathrm{He}^{+},{ }^{12} \mathrm{C}^{+}$and ${ }^{12} \mathrm{C}^{++}$. The starting program will last about 4 years and foresees the study of the carbon burning through the cross section measurement of ${ }^{12} \mathrm{C}+{ }^{12} \mathrm{C}$ processes, such as $\left.{ }^{12} \mathrm{C}\left({ }^{12} \mathrm{C}, \mathrm{p}\right){ }^{23} \mathrm{Na},{ }^{12} \mathrm{C}\left({ }^{12} \mathrm{C}, \alpha\right)\right)^{20} \mathrm{Ne}$ and ${ }^{12} \mathrm{C}\left({ }^{12} \mathrm{C}, \mathrm{n}\right){ }^{23} \mathrm{Mg}$. Carbon Burning is of crucial importance to understand the evolution of stars. In fact, when He burning subsides, the lack of radiation pressure sends the core of the star into gravitational free fall, with the temperature increasing in response to the decrease in gravitational potential energy. This collapse is halted by one of two possible events: the temperature becomes sufficient to ignite the carbon in the star's core or electron degeneracy halts the contraction producing a carbon-oxygen white dwarf. Which of these paths a particular star follows is dependent on whether the ignition temperature for carbon fusion is reached $\left(\sim 5 \times 10^{8} \mathrm{~K}\right)$ or, analogously, whether the critical mass for carbon ignition $\left(\sim 9 \mathrm{M}_{\odot}\right)$ is exceeded prior to electron degeneracy. Thus the end of helium burning marks a branching point in stellar evolution. Lower mass stars will become stable, electrondegenerate white dwarfs, while higher mass stars will enter the quiescent carbon burning phase of their evolution. As a consequence, this limit separates the progenitors of white dwarfs, novae and type Ia supernovae, from those of core-collapse supernovae, neutron stars, and stellar mass black holes. This mass limit also controls the estimations of the expected numbers of these objects in a given stellar population. The rate of the ${ }^{12} \mathrm{C}+{ }^{12} \mathrm{C}$ reaction, which is the trigger of the carbon burning, is a primary input to predict the fate of a star at this branching point. The larger the ${ }^{12} \mathrm{C}+{ }^{12} \mathrm{C}$ rate, the lower the temperature of the carbon burning, with a shorter duration of the $\mathrm{C}$ burning phase. Carbon burning also influences the nucleosynthesis of massive stars. As a matter of fact, the two main channels of this reaction release protons and $\alpha$ particles in a rather hot environment, thus allowing a complex chain of reactions involving nuclei from $\mathrm{C}$ to $\mathrm{Si}$. Some of these reactions, e.g. the ${ }^{13} \mathrm{C}(\alpha, n){ }^{16} \mathrm{O}$ and the ${ }^{22} \mathrm{Ne}(\alpha, n){ }^{25} \mathrm{Mg}$, release neutrons and, in turn, activate the s-process which allows the production of heavy elements (see below). The ${ }^{12} \mathrm{C}+{ }^{12} \mathrm{C}$ rate also affects the outcomes of type Ia supernovae, that play a fundamental role in cosmology, allowing the measurements of distances and of the expansion rates of high redshift galaxies. The understanding of these different phenomena, of primary importance for astrophysics and cosmology, deserves a deeper experimental investigation of the ${ }^{12} \mathrm{C}+{ }^{12} \mathrm{C}$ cross section. As shown in table 1, the initial program with the LUNA-MV accelerator includes the study of the ${ }^{13} \mathrm{C}(\alpha, n){ }^{16} \mathrm{O}$ and ${ }^{22} \mathrm{Ne}(\alpha, n){ }^{25} \mathrm{Mg}$ reactions. These reactions release neutrons and, in turn, activate the s-process which allows the production of heavy elements. Finally, the measurement of the ${ }^{12} \mathrm{C}(\alpha, \gamma){ }^{16} \mathrm{O}$ with LUNA-MV has been considered. This is a key reaction during the stellar phase of helium burning. However the existence of a beam induced background due to the ${ }^{13} \mathrm{C}(\alpha, n){ }^{16} \mathrm{O}$ reaction suggests an inverse kinematic approach, with a ${ }^{12} \mathrm{C}$ ion beam impinging a ${ }^{4} \mathrm{He}$ gas target. 


\section{References}

[1] C. Rolfs and W.S. Rodney: Cauldrons in the cosmos, University of Chicago Press (1988).

[2] C. Iliadis, Nuclear Physics of Stars (Weinheim:Wiley-VCH) (2007).

[3] U. Greife et al, NIM A 350 (1994). Volume 350, 327-337, (1994).

[4] R. Bonetti et al, Phys. Rev. Lett. 82, 5205 (1999).

[5] J.N. Bahcall, M.H. Pinsonneault, Rev. Mod. Phys. 64, 885 (1992).

[6] W.A. Fowler, Nature 238, 24 (1972).

[7] A. Formicola et al, Nucl. Instr. and Meth. A 507609 (2003).

[8] P. Prati et al, Z. Phys. A 350, 171-176 (1994).

[9] H. Costantini et al, Phys. Lett. B 482 43-49 (2000).

[10] M. Aliotta et al, Nucl. Phys. A 690 790-800 (2001).

[11] C. Casella et al, Nuclear Physics A 706 203-216. (2002).

[12] "LUNA proposal 20015-2018", submitted to the LNGS scientific committee.

[13] E. Di Valentino, C. Gustavino et al., Phys. Rev. D 90, 023543 (2014).

[14] D. Bemmerer et al, Phys. Rev. Lett. 97, 122502 (2006).

[15] F. Confortola et al, Phys. Rev. C 75, 065803 (2007).

[16] A. Formicola et al, Phys. Lett. B 591, 61 (2004).

[17] G. Imbriani AA 420 625Đ629 (2004).

[18] G. Imbriani et al, Eur. Phys. J. A, 25 455-466 (2005).

[19] M. Marta et al, Phys. Rev. C 78, 022802 (2008).

[20] A. Lemut et al, Phys. Lett. B 634483 (2006).

[21] B.Limata et al, Phys. Rev. C 82, 015801 (2010).

[22] F. Strieder et al, Phys. Lett. B 7070 60-65 (2012).

[23] O.Straniero et al, The Astrophysical Journal, 763:100 (10pp) (2013).

[24] D.Bemmerer et al, J. Phys. G: Nucl. Part. Phys. 36045202 (2009).

[25] A.Caciolli et al, AA 533, A66 (2011).

[26] D.A. Scott et al, Phys. Rev. Lett. 109, 202501 (2012).

[27] A. Di Leva et al, Phys. Rev. C 89, 015803 (2014).

[28] R. De Palo et al, Phys. Rev. C 94, 055804 (2016).

[29] F. Ferraro et al, Eur. Phys. J. A 5444 (2018).

[30] C.G. Bruno et al, Phys. Rev. Lett. 117, 142502 (2016).

[31] M.Lugaro et al, Nature Astronomy 1, 0027 (2017).

[32] M. Anders et al, PRL 113, 042501 (2014).

[33] D. Trezzi et al, Astroparticle Physics 89, 57-65 (2017).

[34] The LUNA Collaboration, LUNA-MV Proposal 2018-2022. INFN/17-19/LNGS (2017). 